\title{
Reasons for termination of pregnancy in mid-trimester: A single-centre experience
}

\section{Dear Editor,}

This is a retrospective study conducted on 118 women who underwent mid-trimester pregnancy termination (MTPT) at 12-24 weeks' gestational age (GA) at a tertiary obstetric unit in the National University Hospital, Singapore.

There are limited data and studies examining MTPT across various institutions worldwide, which legalise abortions, including Singapore. Despite the accessibility, ease and safety of performing first trimester termination of pregnancy (TOP), there remains a small group of women who opt for MTPT for fetal anomalies. Data collated from our institution on 118 women who underwent MTPT from 15 December 2015 to 12 December 2018 demonstrated that the main indications for MTPT were social reasons $(49.2 \%)$ and fetal anomalies/maternal reasons $(50.8 \%)$. Other outcome measures included maternal age, booking body mass index, marital status, smoking, parity, history of previous TOP, previous caesarean sections, number of cycles of vaginal prostaglandins used before successful MTPT, need for surgical evacuation of uterus (SEU) post-MTPT and post-MTPT contraception uptake.

More than half of the women (30/58, 51.7\%) underwent MTPT for social reasons before GA 16 weeks $(P=0.0002)$, while $9(15.5 \%)$ were $\mathrm{GA} \geq 20$ weeks. In contrast, 31 (52.5\%) women who underwent MTPT for fetal anomalies were $\mathrm{GA} \geq 20$ weeks $(P<0.0001)$. Women who required TOP for fetal anomaly tend to present later as fetal anomalies were picked-up after their antenatal ultrasound scans, typically performed at GA 20-22 weeks. They were more likely to be married $(96.6 \%)$ and older (mean age 33.6 years) compared to women who required TOP for social reasons (mean age 28.0 years, $P<0.0001)$.

All MTPTs were associated with known risks of retained products of conception and a 5-30\% risk of requiring SEU. ${ }^{1}$ Increased risk of complications of SEU in second trimester abortions ${ }^{2-4}$ included risks of heavy bleeding, cervical trauma, Asherman's syndrome and cervical incompetence with increasing GA. ${ }^{5} \mathrm{~A}$ study conducted from 2005-2009 in Singapore focusing on teenage and late abortions (defined as TOP in mid-trimester) examined risk factors for late presentation for abortions. Important risk factors identified for late TOP in the second trimester were women who were less than 20 years old, of Malay ethnicity, single, nulliparous, had a history of prior abortions and no prior contraceptive usage. ${ }^{6}$ Understanding the demographics of women who underwent MTPT for social reasons and the possible risks associated with post-MTPT SEU would allow early recognition and appropriate riskstratification for these women pre-MTPT, as well as to proffer post-MTPT contraceptive advice to prevent repeated abortions.

Women could still choose to undergo MTPT for fetal anomalies and we had evaluated the effectiveness of our medical regimen for MTPT to reduce the risks of SEU. Successful MTPT was defined as expulsion of fetus with one cycle of misoprostol with or without SEU. Utilising the misoprostol-only regime for MTPT at our unit, we discovered that MTPT achieved a success rate of $85.6 \%$ and was similarly efficacious in women who had previous caesarean section (success rate $80.6 \%$ ). This regimen was in line with the evidenced-based regimen recommended by the UK Royal College of Obstetrics and Gynaecologists for termination of pregnancies between 13 and 22 weeks when mifepristone is not available. ${ }^{7}$

We had also gathered data on post-MTPT contraception uptake, which was surprisingly low and less than 30\% $(35 / 118)$ of women took up contraception post-MTPT. Among them, only 1 woman who underwent MTPT for fetal anomalies decided to take up contraception post-MTPT. We expected a higher uptake of any choice of contraception for women who needed SEU, as opportunistic insertion of a long-acting reversible contraception could be performed simultaneously when the women were under general anaesthesia for SEU. However, we did not observe any differences in contraception uptake in women who underwent SEU versus those women who did not require SEU.

No study to date has examined the profile of women in Singapore who underwent MTPT, the MTPT regimen employed, and the rates of SEU post-MTPT. Our data demonstrated the efficacy of our MTPT 
regimen and highlighted the gaps in post-abortion care, which needed to be addressed urgently. More data on post-abortion care and reproductive sequelae in women who underwent MTPT were required as most women were lost to follow-up. Complications such as infection causing pelvic inflammatory disease, Asherman's syndrome, cervical incompetence or infertility after MTPT might be underestimated. Furthermore, given the low uptake of contraception in women who underwent MTPT for social reasons, concerted efforts by nursing, medical and allied healthcare professionals would be pertinent to ensure that these women understood the consequences of unplanned pregnancies and abortions, so as to increase the utility of contraception.

We should recognise that late abortions could be potentially debilitating to a woman's sexual and reproductive health. More importantly, in countries and territories where abortions are legal, it is imperative to reduce or even prevent late abortions due to social reasons by provision of comprehensive pre- and postabortion care and family-planning services. This will ensure improved contraceptive uptake and full compliance with contraceptive usage.

Table 1. Characteristics of women undergoing MTPT between 2015 and 2018

\begin{tabular}{|c|c|c|}
\hline Variable & Definition & $\begin{array}{l}\text { Study population } \mathrm{N}=118 \\
\text { n (\%) }\end{array}$ \\
\hline \multirow[t]{3}{*}{ Gestational age at MTPT } & 12-16 weeks & $40(33.8)$ \\
\hline & 17-20 weeks & $39(33.1)$ \\
\hline & 21-24 weeks & $39(33.1)$ \\
\hline \multirow[t]{2}{*}{ Maternal age (years) } & $<25$ & $24(20.3)$ \\
\hline & $\geq 25$ & $94(77.7)$ \\
\hline \multirow[t]{4}{*}{ Ethnicity } & Chinese & $45(38.1)$ \\
\hline & Malay & $29(24.6)$ \\
\hline & Indian & $30(25.4)$ \\
\hline & Others & $14(11.9)$ \\
\hline \multirow[t]{2}{*}{ Marital status } & Single & $28(23.7)$ \\
\hline & Married & $90(76.3)$ \\
\hline \multirow[t]{2}{*}{ Parity } & Primigravida & $44(37.3)$ \\
\hline & Multigravida & $74(62.7)$ \\
\hline \multirow[t]{2}{*}{ Previous caesarean section } & 0 & $87(73.7)$ \\
\hline & $\geq 1$ & $31(26.3)$ \\
\hline \multirow[t]{2}{*}{ Previous TOP } & 0 & $97(82.2)$ \\
\hline & $\geq 1$ & $21(17.8)$ \\
\hline \multirow[t]{3}{*}{ Indication for TOP } & Social & $58(49.2)$ \\
\hline & Fetal anomaly & $59(50)$ \\
\hline & Maternal reasons & $1(0.8)$ \\
\hline \multirow[t]{2}{*}{ BMI $\left(\mathrm{kg} / \mathrm{m}^{2}\right)$} & $<23$ & $46(39.0)$ \\
\hline & $\geq 23$ & $72(61.0)$ \\
\hline \multirow[t]{3}{*}{ Smoking status } & Smokers & $15(12.7)$ \\
\hline & Non-smokers & $39(33.1)$ \\
\hline & Unknown & $64(54.2)$ \\
\hline
\end{tabular}

BMI: body mass index; MTPT: mid-trimester pregnancy termination; TOP: termination of pregnancy 


\section{REFERENCES}

1. Mentula $\mathrm{M}$, Niinimäki $\mathrm{M}$, Suhonen $\mathrm{S}$, et al. Immediate adverse events after second trimester medical termination of pregnancy: results of a nationwide registry study. Hum Reprod 2011; 26:927-32.

2. Punjyashthira A, Pongrojpaw D, Suwannarurk K, et al. The effectiveness of sublingual or oral administration of misoprostol for cervical ripening before manual vacuum aspiration in first trimester termination of pregnancy: randomized controlled trial. J Med Assoc Thai 2014; 97:1009-15.

3. Meirik O, My Huong NT, Piaggio G, et al. Complications of first-trimester abortion by vacuum aspiration after cervical preparation with and without misoprostol: a multicentre randomised trial. Lancet 2012; 379:1817-24.

4. Kerns J, Steinauer J. Management of postabortion hemorrhage: release date November 2012 SFP Guideline \#20131. Contraception 2013;87:331-42.

5. Second-trimester abortion. Practice Bulletin No. 135. American College of Obstetricians and Gynecologists. Obstet Gynecol 2013;121:1394-406.
6. Lim L, Wong H, Yong E, et al. Profiles of women presenting for abortions in Singapore: focus on teenage abortions and late abortions. Eur J Obstet Gynecol Reprod Biol 2012;160:219-22.

7. Hammond C. Recent advances in second-trimester abortion: an evidence-based review. Am J Obstet Gynecol 2009; 200:347-56.

Valerie Low, ${ }^{1} M B B S$, Soe-na Choo, ${ }^{2}{ }_{M b B c h ~ B a O}$, Zhongwei Huang, ${ }^{2,3}$ PhD (Oxon)

${ }^{1}$ Yong Loo Lin School of Medicine, National University of Singapore, Singapore

${ }^{2}$ Department of Obstetrics and Gynaecology, National University Hospital, Singapore

${ }^{3}$ Institute of Molecular and Cell Biology, Agency for Science, Technology and Research, Singapore

Correspondence: Dr Zhongwei Huang, Department of Obstetrics and Gynaecology, NUHS Tower Block Level 12, E Lower Kent Ridge Road, Singapore 119228.

Email: obgzwh@nus.edu.sg 